\title{
Optical absorption and fluorescence studies on imidazolium ionic liquids comprising the bis(trifluoromethanesulphonyl)imide anion
}

\author{
ANIRUDDHA PAUL and ANUNAY SAMANTA* \\ School of Chemistry, University of Hyderabad, Hyderabad 500046 \\ e-mail: assc@uohyd.ernet.in
}

MS received 18 April 2006; revised 27 June 2006

\begin{abstract}
Optical absorption and fluorescence behaviour of two rigorously purified imidazolium ionic liquids, 1-butyl-3-methylimidazolium bis(trifluoromethanesulfonyl)imide and 1-ethyl-3-methylimidazolium bis(trifluoromethanesulfonyl)imide are studied in the neat condition and in solution. Non-negligible absorption in the UV region with a long tail extending into the visible region is the main feature of the absorption. Excitation wavelength-dependent two-component fluorescence characterizes the emission behaviour of these liquids. That ion association gives rise to the long absorption tail and shifting fluorescence maximum, which appears to be common to most of the imidazolium ionic liquids, is evident from the effect of the conventional solvents.
\end{abstract}

Keywords. Imidazolium ionic liquids; UV-Vis absorption; excitation wavelength-dependent fluorescence; red-edge effect; solvent effect.

\section{Introduction}

Room-temperature ionic liquids based on imidazolium cations have attracted considerable attention in recent years because of the possibility that these substances can serve as environmentally benign media, in place of volatile organic compounds. ${ }^{1-3}$ Apart from characterisation of their properties, the utility of these novel substances in various applications, such as synthesis, catalysis, electrochemical and photophysical studies, has already been demonstrated. ${ }^{1-21}$ The most attractive property of these ionic liquids from the point of view of their environmentally benign nature is their negligible vapor pressure. Wide liquidus, thermal stability, high ionic conductivity, miscibility with other solvents and recyclable nature are some of the other properties that make these liquids suitable as solvents for various applications. These are often described as 'designer solvents', as it is possible to design an ionic liquid with the desired properties using appropriate combinations of cationic and anionic constituents.

Since application of the ionic liquids as media of photophysical studies depends on how transparent these substances are in the optical region, we have

\footnotetext{
*For correspondence
}

recently characterised the UV-visible absorption and fluorescence behaviour of a few imidazolium ionic liquids. ${ }^{18,19}$ Contrary to the common perception that these are optically transparent substances, ${ }^{8,17,20,21}$ our results show that $\left[\mathrm{PF}_{6}\right]^{-}$and $\left[\mathrm{BF}_{4}\right]^{-}$based imidazolium ionic liquids have non-negligible absorption in the UV region, with the absorption tail extending well into the visible region. These studies have also revealed the interesting fluorescence behaviour of the imidazolium ionic liquids. ${ }^{18,19}$ The fluorescence maxima of previously studied ionic liquids have been found to be strongly dependent on the excitation wavelength. $^{18,19}$ In order to convincingly demonstrate that the absorption and emission features, which have been observed for a few ionic liquids, are common to most of the imidazolium ionic liquids, it is necessary that further studies be undertaken on

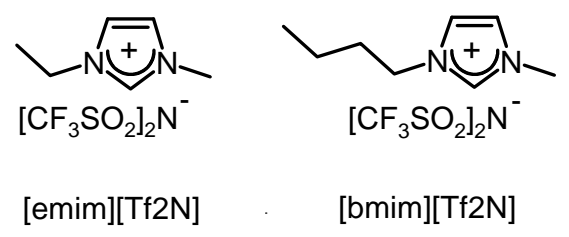

Chart 1. Chemical formula and abbreviations of the ionic liquids. 
various imidazolium ionic liquids. Further studies are also necessary for an understanding of the origin of the peculiar absorption and fluorescence behaviour of these widely used substances. With this view in mind, we have undertaken the present study in which we focus on two relatively less viscous ionic liquids, 1-butyl-3-methylimidazolium bis(trifluoromethanesulfonyl)imide and 1-ethyl-3-methylimidazolium bis(trifluoromethanesulfonyl)imide, abbreviated as $[\mathrm{bmim}]\left[\mathrm{Tf}_{2} \mathrm{~N}\right]$ and $[\mathrm{emim}]\left[\mathrm{Tf}_{2} \mathrm{~N}\right]$ respectively. Since the unusually long absorption tail and shifting nature of the fluorescence have been attributed to the associated nature of the constituent ions of the imidazolium ionic liquids, ${ }^{18,19}$ we felt that the absorption and fluorescence behaviour of relatively less viscous $[\mathrm{bmim}]\left[\mathrm{Tf}_{2} \mathrm{~N}\right]$ and $[\mathrm{emim}]\left[\mathrm{Tf}_{2} \mathrm{~N}\right]$ might throw information on how important the ion association is in these relatively less viscous media. For this reason, we have also examined the effect of the conventional solvents on the absorption and fluorescence behaviour of these two ionic liquids.

\section{Experimental}

\subsection{Synthesis and purification of [bmim] $\left[\mathrm{Tf}_{2} \mathrm{~N}\right]$ and [emim] $\left[\mathrm{Tf}_{2} \mathrm{~N}\right]$}

The two ionic liquids were synthesized according to the reported procedure from the corresponding chloride salts. ${ }^{8}$ The chloride salt and $\mathrm{LiTf}_{2} \mathrm{~N}$ were dissolved in equimolar amounts in doubly distilled de-ionized water and the mixture was stirred for at least $18 \mathrm{~h}$, first at room temperature and then at $50-60^{\circ} \mathrm{C}$, under nitrogen atmosphere. After cooling the mixture, the viscous lower (hydrophobic) part was separated and washed with water several times, until no trace of halide was detected in the wash-liquid by the $\mathrm{AgNO}_{3}$ test. Subsequently, the viscous liquid was washed with hexane a few times to remove organic impurities (if any) that may contribute to the absorption and fluorescence behaviour of the ionic liquid. Following this, the ionic liquid was diluted with dry DCM (freshly distilled), quickly filtered through a celite plug and then DCM solution was evaporated under reduced pressure to obtain the neat ionic liquid. This was then dried under high vacuum (with heating at $60^{\circ} \mathrm{C}$ for $[\mathrm{bmim}]\left[\mathrm{Tf}_{2} \mathrm{~N}\right]$ ) for at least $10 \mathrm{~h}$ to minimize the water content. The ionic liquids thus prepared were characterized by NMR and IR spectroscopic techniques. Freshly prepared ionic liquid was used for the studies described here.

\subsection{Instrumentation}

Absorption and steady-state fluorescence spectra were recorded on a UV-Vis spectrophotometer (Cary100, Varian) and a spectrofluorimeter (FluoroLog-3, Jobin Yvon) respectively. The fluorescence spectra were corrected for instrumental response. Time-resolved fluorescence measurements were carried out using a time-correlated single-photon counting (TCSPC) spectrometer $(5000, \mathrm{IBH})$. Diode laser $\left(\lambda_{\mathrm{exc}}=374 \mathrm{~nm}\right.$, FWHM $=65 \mathrm{ps}$ ) was used as the excitation source and an MCP photomultiplier (Hamamatsu R3809U50) as the detector. The lamp profile was recorded by placing a scatterer (dilute solution of Ludox in water) in place of the sample. Decay curves were analysed by nonlinear least-squares iteration procedure using IBH DAS6 (Version 2.2) decay analysis software. ${ }^{1} \mathrm{H}$ NMR spectra were recorded on a Bruker Avance $400 \mathrm{MHz}$ NMR spectrometer at ambient temperature using tetramethylsilane (TMS) as internal standard.

\section{Results and discussion}

Since highly pure ionic liquids are required for the optical measurements, special care was taken for the purification of the two ionic liquids studied here. In addition to the removal of the halides by repeated washing with water, the two liquids were washed several times with $n$-hexane to remove any organic impurity that may contribute to the absorption and

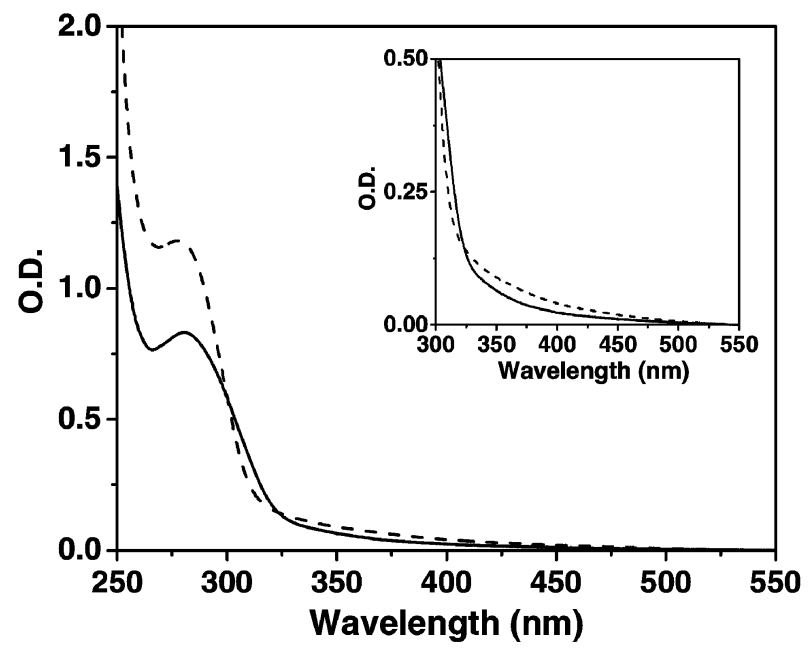

Figure 1. Absorption spectra of neat $[\mathrm{bmim}]\left[\mathrm{Tf}_{2} \mathrm{~N}\right](-)$ and $\left[\right.$ emim] $\left[\mathrm{Tf}_{2} \mathrm{~N}\right](---)$, as obtained using a $1-\mathrm{cm}$ pathlength cuvette. The inset shows the tail of the absorption band more clearly. 

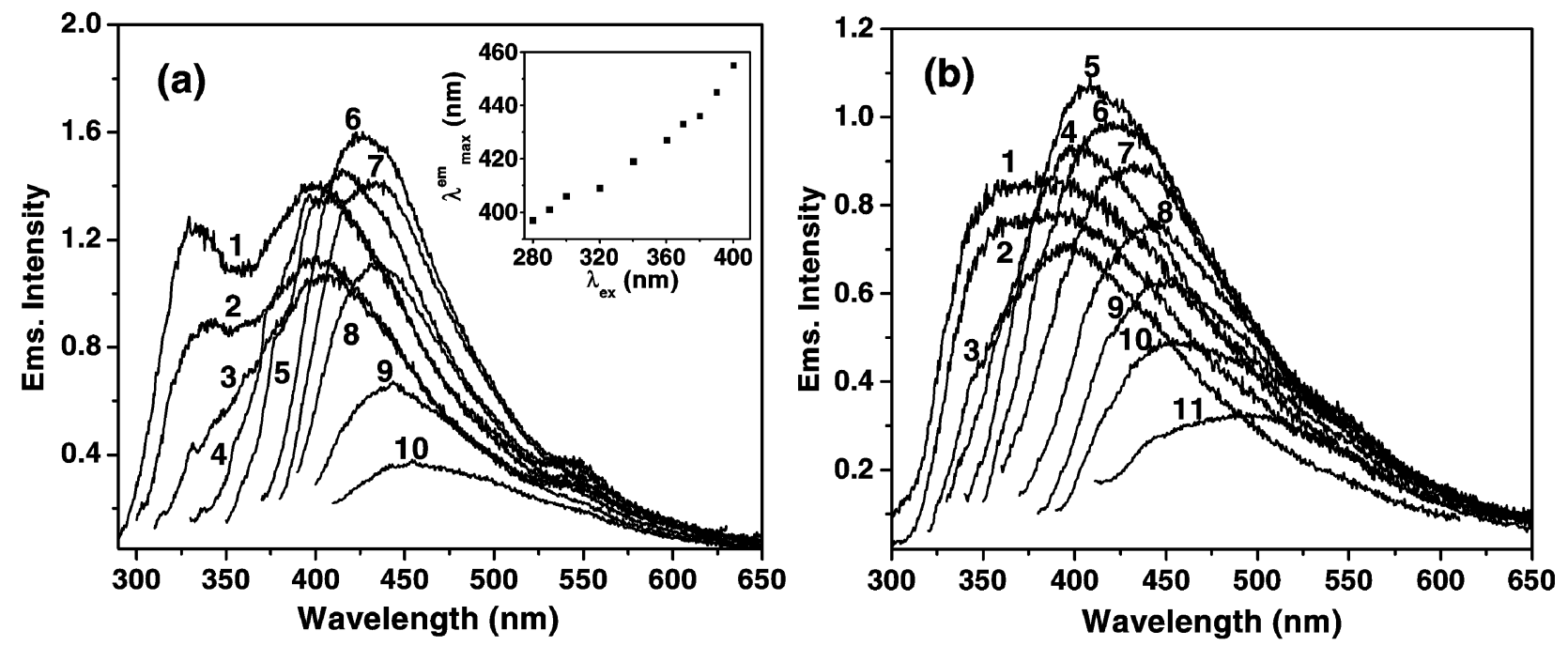

Figure 2. Excitation wavelength-dependent emission behaviour of neat (a) $[\mathrm{bmim}]\left[\mathrm{Tf}_{2} \mathrm{~N}\right]$ and $(\mathbf{b})\left[\mathrm{emim}^{\mathrm{b}}\right]\left[\mathrm{Tf}_{2} \mathrm{~N}\right]$. The excitation wavelengths in (a) for the spectra labelled 1 to 10 are 280, 290, 300, 320, 340, 360, 370, 380, 390 and $400 \mathrm{~nm}$ respectively and in (b) for spectra labelled 1 to 11 are 280, 290, 310, 320, 330, 340, 350, 360, 370, 380 , and $400 \mathrm{~nm}$ respectively.

emission in the region of interest. The absorption spectra of neat $[\mathrm{bmim}]\left[\mathrm{Tf}_{2} \mathrm{~N}\right]$ and $[\mathrm{emim}]\left[\mathrm{Tf}_{2} \mathrm{~N}\right]$ are shown in figure 1 . As can be seen, the absorption features of the two imidazolium ionic liquids are very similar. The absorbance due to these liquids in a cuvette of $1 \mathrm{~cm}$ pathlength varies between 0.58 and 0.6 at $300 \mathrm{~nm}$ and 0.06 and 0.09 at $350 \mathrm{~nm}$. These absorbance values are comparable to those observed for the previously studied ionic liquids, $[\mathrm{bmim}]\left[\mathrm{BF}_{4}\right], \quad[\mathrm{emim}]\left[\mathrm{BF}_{4}\right]$ and $[\mathrm{bmim}]\left[\mathrm{PF}_{6}\right] .{ }^{18,19}$ The hump around $280 \mathrm{~nm}$ appears to be more prominent compared to the previously studied ionic liquids. A long absorption tail, which was observed previously for the other ionic liquids, is also clearly visible for the present systems.

Both $[\mathrm{bmim}]\left[\mathrm{Tf}_{2} \mathrm{~N}\right]$ and $[\mathrm{emim}]\left[\mathrm{Tf}_{2} \mathrm{~N}\right]$ are found to be fluorescent. The fluorescence spectra of these liquids (figure 2) consist of two components, which are more clearly resolved in the case of [bmim] $\left[\mathrm{Tf}_{2} \mathrm{~N}\right]$. When excited at $280 \mathrm{~nm}$, fluorescence maxima $\left(\lambda_{\max }^{\mathrm{flu}}\right)$ are observed at around 330 and $400 \mathrm{~nm}$ for [bmim] $\left[\mathrm{Tf}_{2} \mathrm{~N}\right]$. Fluorescence behaviour is found to be strongly dependent on the excitation wavelength. On increasing the excitation wavelength from 280 to $320 \mathrm{~nm}$, the short-wavelength emission component gradually vanishes. When the excitation wavelength is increased further, $\lambda_{\max }^{\text {flu }}$ of the surviving longcomponent starts shifting towards red with progressive decrease of the intensity. No limiting value of $\lambda_{\max }^{\mathrm{flu}}$ is observed and the shift of $\lambda_{\max }^{\mathrm{flu}}$ is observable as long as it is possible to record a fluorescence spectrum. A plot of the variation of $\lambda_{\max }^{\text {flu }}$ with the excitation wavelength $\left(\lambda_{\text {exc }}\right)$ is shown in the inset of figure 2a. In the case of [emim] $\left[\mathrm{Tf}_{2} \mathrm{~N}\right]$, the situation is slightly different in the sense that the two components are not clearly resolved. Instead, a broad emission band, stationary in nature, is observed for short excitation wavelengths $(280-310 \mathrm{~nm})$. That this broad band consists of two components is evident when the excitation wavelength is increased. An increase of the excitation wavelength first leads to weakening of the intensity in the short wavelength region of the spectrum, resulting in decrease in the width of the spectrum. A further increase of the excitation wavelength gives rise to shift of the fluorescence maximum.

We have also attempted to quantify the fluorescence efficiency of these ionic liquids. However, because of their sensitivity to the excitation wavelength, fluorescence quantum yield is dependent on the excitation wavelength. An idea of how fluorescent these liquids are can however be obtained for specific excitation wavelengths. The quantum yield $\left(\phi_{f}\right)$ of $[$ bmim $]\left[\mathrm{Tf}_{2} \mathrm{~N}\right]$ is estimated as 0.008 (4-aminophthalimide as reference compound, $\phi_{f}=0.1$ in methanol $)^{22}$ corresponding to the excitation wavelength of $360 \mathrm{~nm}$. The $\phi_{f}$ value of $[\mathrm{emim}]\left[\mathrm{Tf}_{2} \mathrm{~N}\right]$ is estimated as 0.004 (for $\lambda_{\text {exc }}=350 \mathrm{~nm}$ ). These values 
are comparable to the fluorescence efficiency of the other imidazolium ionic liquids. ${ }^{18,19}$

Fluorescence decay behaviour of the two ionic liquids has been studied by exciting each sample at $374 \mathrm{~nm}$ and monitoring the emission around $550 \mathrm{~nm}$. The decay profiles are found to be complex in nature, consisting of multiple components. A tri-exponential fit to the decay profiles is however found satisfactory. The major component of the decay (50-68\%) comprises a lifetime of $350-500 \mathrm{ps}$. Two other long components with lifetimes $2 \cdot 5-2 \cdot 8 \mathrm{~ns}(20-35 \%)$ and 9.4-9.6 ns (12-15\%) are also observed. Typical fluorescence decay behaviour, monitored at $550 \mathrm{~nm}$, along with the best fit are shown in figure 3 .

The results show that the absorption and fluorescence properties of these two ionic liquids are indeed quite similar to those of other imidazolium ionic liquids. Since the long absorption tail and shifting nature of the fluorescence spectra have previously been attributed to the presence of various energetically different associated forms of the cationic component in the ground state and the inefficiency of the energy transfer between these forms, ${ }^{18,19}$ the present observation is not surprising. However, since the anionic component plays a key role in the association of the cationic components (the imidazolium ions), by directly getting involved in the association and also by indirectly influencing the association by changing the viscosity and polarity of the media, some variation in the absorption and fluorescence properties

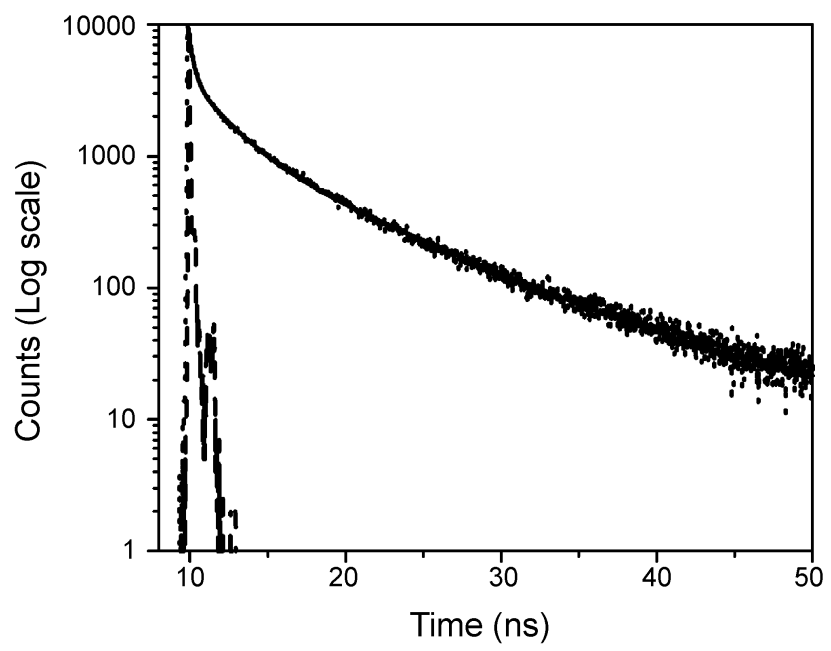

Figure 3. Fluorescence decay profile of $[\mathrm{bmim}]\left[\mathrm{Tf}_{2} \mathrm{~N}\right]$ as monitored at $550 \mathrm{~nm}$. The experimental decay curve is shown as a dotted line and the instrument profile as a dashed line. The solid line is the triexponential fit to the decay curve. for different imidazolium ionic liquids is expected. However, the present results suggest that this variation is not very significant.

We have also studied the influence of conventional solvents on the absorption and fluorescence behaviour of ionic liquids with a view to obtaining support in favour of the associated nature of the absorbing and fluorescing species. Tetrahydrofuran and acetonitrile have been used for this purpose because of their high solubility in these ionic liquids. Figure 4 depicts the fluorescence spectra of tetrahydrofuran and acetonitrile solutions $(0 \cdot 3-0 \cdot 4 \mathrm{M})$ of $[\mathrm{bmim}]\left[\mathrm{Tf}_{2} \mathrm{~N}\right]$, measured for various excitation wavelengths. The shifting nature of the long wavelength emission band is clearly observable in these fairly concentrated solutions of the ionic liquids. The fluorescence excitation spectra corresponding to the shifting emission band, whose behaviour is similar to those observed previously for other ionic liquids, ${ }^{19}$ suggest that the different emission peaks are due to species that absorb in the tail portion of the absorption band.

Two other features of the emission spectra are noteworthy. First, the intensity of the long-wavelength emission band relative to that of the shortwavelength band $\left(I_{\text {long }} / I_{\text {short }}\right)$ in these solutions is lower than that in neat liquids. Moreover, with decrease in the concentration of the ionic liquid, the relative intensity of the long-wavelength band decreases. It can be seen that the long-wavelength emission component ultimately vanishes at high dilution $(\sim 0.004 \mathrm{M})$. This observation is consistent with the assignment that the short-wavelength component is due to unassociated imidazolium ion and the longwavelength component is due to the various associated forms of the imidazolium ion. A lower intensity of the long-wavelength emission band in solutions is obviously a reflection of the fact that dilution of the ionic liquids leads to breaking of some of the associated structures. Second, the long-wavelength band is blue-shifted in the relatively less polar solvent, tetrahydrofuran. In acetonitrile, which is almost as polar as the neat ionic liquids, the peak position appears at $400 \mathrm{~nm}$, which is the same as that obtained in neat ionic liquid. On the other hand, in less polar tetrahydrofuran, the peak appears at $385 \mathrm{~nm}$. This observation seems to indicate that the excited state of the associated species is more polar than the ground state.

It must be recognized that it is extremely difficult to comment on the exact nature of the associated forms of the imidazolium ions responsible for the optical absorption and emission behaviour, in parti- 

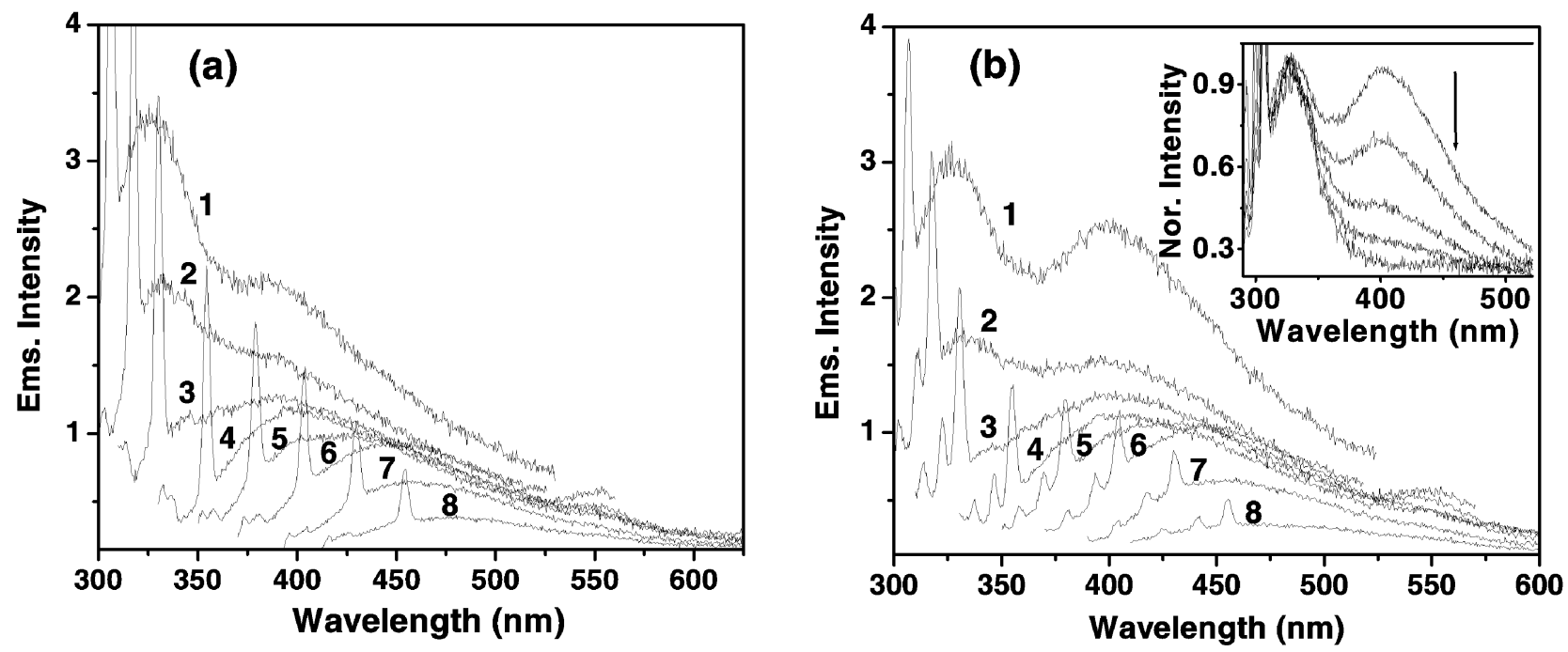

Figure 4. Excitation wavelength dependent emission behaviour of tetrahydrofuran (a) and acetonitrile (b) solutions of $[\mathrm{bmim}]\left[\mathrm{Tf}_{2} \mathrm{~N}\right](0 \cdot 35-0 \cdot 4 \mathrm{M})$. In both cases, $\lambda_{\text {exc }}(\mathrm{nm})=280$ (1), 290 (2), 300 (3), 320 (4), 340 (5), 360 (6), 380 (7) and 400 (8). The sharp peaks are Raman lines. The inset in figure (b) shows that dilution of [bmim] $\left[\mathrm{Tf}_{2} \mathrm{~N}\right]$ by acetonitrile leads to gradual disappearance of the long-wavelength component. The concentrations of $[\mathrm{bmim}]\left[\mathrm{Tf}_{2} \mathrm{~N}\right]$ in decreasing order of the intensities at $400 \mathrm{~nm}$ are $0 \cdot 4,0 \cdot 2,0 \cdot 1,0.04$ and $0.004 \mathrm{M}$ respectively. The spectra shown in the inset are normalized at the short wavelength peak $(\sim 326-327 \mathrm{~nm})$.

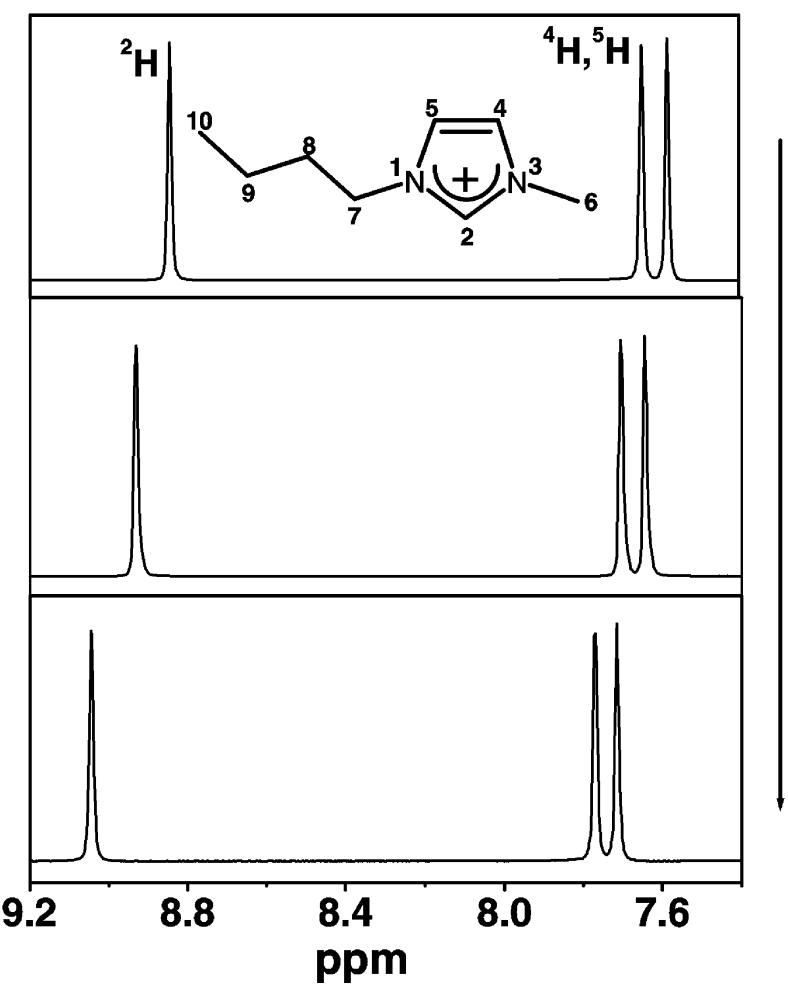

Figure 5. Concentration dependence of the ${ }^{1} \mathrm{H}-\mathrm{NMR}$ spectra of $[\mathrm{bmim}]\left[\mathrm{Tf}_{2} \mathrm{~N}\right]$ in acetone- $d^{6}$ (only the peaks corresponding to the ring protons are shown). The concentrations of $[\mathrm{bmim}]\left[\mathrm{Tf}_{2} \mathrm{~N}\right]$ from top to bottom are 1.65 $\mathrm{M}, 0 \cdot 84 \mathrm{M}$ and $0 \cdot 17 \mathrm{M}$ respectively. The atom numbering and the peak assignments are also indicated in the figure. cular, the long absorption tail and the shifting fluorescence band. This is because of the fact that associated forms of different kinds have been indicated in previous studies. ${ }^{4,23-29}$ Evidence has been obtained for cation-cation $\pi$-interaction, hydrogenbonding interaction, cation-anion electrostatic interaction etc. through conductometric, NMR, X-ray scattering, Raman spectroscopy and FAB-MS measurements. ${ }^{4,23-29}$ However, since hydrogen-bonding interaction is believed to be unimportant in the case of $\left[\mathrm{Tf}_{2} \mathrm{~N}\right]$ ionic liquids ${ }^{8}$, the other interactions appear to be more important in the present cases. We found that the NMR signals of all the protons connected to the imidazolium ring in $[\mathrm{bmim}]\left[\mathrm{Tf}_{2} \mathrm{~N}\right]$ (in acetone$\left.d^{6}\right)$ shift to higher $\delta$-values with decreasing concentrations of the ionic liquids (figure 5). A similar observation for $[\mathrm{emim}]\left[\mathrm{Tf}_{2} \mathrm{~N}\right]$ and other fluorous ionic liquids has previously been explained considering the shielding effect of the $\pi$-electrons of an imidazolium ring on the protons of another ring immediately (or somewhat horizontally shifted) above or beneath it and pertubation of this stacking by the intervening solvent molecules. ${ }^{8}$ Hence, it is apparent that $\pi$ stacked dimers or oligomers could be the associated structures that contribute to the interesting absorption and fluorescence behaviour of these liquids. However, since the nature of the association could be significantly different for different imidazolium ionic liquids, 
other forms of association contributing to the absorption and fluorescence characteristics cannot be ruled out.

\section{Conclusion}

Electronic absorption and fluorescence behaviour of two less viscous imidazolium ionic liquids based on $\mathrm{Tf}_{2} \mathrm{~N}$ anion is quite similar to that of the other imidazolium ionic liquids. The results unequivocally establish that the absorption and fluorescence due to these liquids in the UV-visible region cannot be ignored while studying the fluorescence response of solute molecules in these media. It appears that $\pi$-stacked dimers and oligomers of the imidazolium ions could be the associated species responsible for the interesting absorption and fluorescence behaviour of the imidazolium ionic liquids. That the imidazolium ions in the associated structures are held by weak interactions is evident from the fact that moderately polar conventional solvents are capable of breaking these structures.

\section{Acknowledgement}

This work is supported by the Department of Science and Technology (DST), Government of India and Council of Scientific and Industrial Research (CSIR). We also thank the UPE Programme of the University Grants Commission (UGC) for providing some of the instrumental facilities. AP thanks CSIR for a fellowship.

\section{References}

1. Welton T 1999 Chem. Rev. 992071

2. Dupont J, de Suza R F and Suarez P A Z 2002 Chem. Rev. 1023667

3. Sheldon R 2001 Chem. Commun. 2399

4. Tokuda H, Hayamizu K, Ishii K, Susan M A B H and Watanabe M 2004 J. Phys. Chem. B108 16593

5. Aki S N V K, Brennecke J F and Samanta A 2001 Chem. Commun. 413
6. Billard I, Moutiers G, Labet A, El Azzi A, Gaillard C, Mariet C and Lutzenkirchen K 2003 Inorg. Chem. 421726

7. Carmichael A J and Seddon K R 2000 J. Phys. Org. Chem. 13591

8. Bonhote P, Dias A P, Papageorgiou N, Kalyanasundaram K and Gratzel M 1996 Inorg. Chem. 35 1168

9. Karmakar R and Samanta A 2002 J. Phys. Chem. A106 6670

10. Ingram J A, Moog R S, Ito N, Biswas R and Maroncelli M 2003 J. Phys. Chem. B107 5926

11. Chakrabarty D, Hazra P, Chakraborty A, Seth D and Sarkar N 2003 Chem. Phys. Lett. 381697

12. Karmakar R and Samanta A 2003 Chem. Phys. Lett. 376638

13. Skrzypczak A and Neta P 2003 J. Phys. Chem. A107 7800

14. Choudhury A R, Winterton N, Steiner A, Cooper A I and Johnson K A 2005 J. Am. Chem. Soc. 12716792

15. Baker S N, Baker G A, Kane M A and Bright F V 2001 J. Phys. Chem. B105 9663

16. Mandal P K, Sarkar M and Samanta A 2004 J. Phys. Chem. A108 9048

17. Muldoon M J, McLean A J, Gordon C M and Dunkin I R 2001 Chem. Commun. 2364

18. Paul A, Mandal P K and Samanta A 2005 J. Phys. Chem. B109 9148

19. Paul A, Mandal P K and Samanta A 2005 Chem. Phys. Lett. 402375

20. Huddleston J G, Willauer H D, Swatlowski R P, Visser A E and Rodgers R D 1998 Chem. Commun. 1765

21. Lancaster N L, Salter P A, Welton T and Young G B 2002 J. Org. Chem. 678855

22. Soujanya T, Krishna T S R and Samanta A $1992 J$. Phys. Chem. 968544

23. Urahata S M and Ribeiro M C C 2004 J. Chem. Phys. 1201855

24. Del Popolo M G and Voth G A 2004 J. Phys. Chem. B108 1744

25. Margulis C J, Stern H A and Berne B J 2002 J. Phys. Chem. B106 12017

26. Mele A, Tran C and Lacerda S H D P 2003 Angew. Chem., Int. Ed. 424364

27. Headley A D and Jackson M N 2002 J. Phys. Org. Chem. 1552

28. Hardacre C, Holbrey J D, McMath S E J, Bowron D T and Soper A K 2003 J. Chem. Phys. 118273

29. Hardacre C, Holbrey J D, McMath S E J, Nieuwenhuyzen M, Bowron D T and Soper A K $2003 \mathrm{~J}$. Phys.: Condens. Matter 15 S159 